\title{
Alignment of the SLC Final Focus System * Using Beam Orbits
}

\author{
Y. Chao, F. LeDiberder, P. Burchat, W. Kozanecki, N. Toge \\ Stanford Linear Accelerator Center, * \\ Stanford, California 94309
}

\section{INTRODUCTION}

Beam based alignment is being routinely applied in the SLC Final Focus and has proved to be a very useful tool for determining the quality of the zeroth order orbit as defined by various beam line elements. Given the stringent requirement on the beam quality at the interaction point (IP), a well aligned beam line is essential in that it minimizes the confusion which would otherwise arise in the higher order optics, the demand called on the correciors which also serve as optical knobs, and the problem associated with the background radiation. In the SLC final focus we have been relying on an interplay betwcen the field survey (mechanical alignment) and the orbit anatysis (beam based alignment) to achicve this purpose. Mechanical alignment gencrally provides coordinate information of various beam line elements and offset values inferred from these data and the model of the beam line. Beam based alignment is done mainly by recording the beam arbit under controlled experiment where optical elements or orbit conditions are varied. Due to the complexity of the beamline layout and special power supply configuration in the SLCFinal Focus, the latcer method is useful only when coupled with off-line analysis which disentangles the data taken at each measurement. In this report we describe the techniques used and the underlying principle, the procedure as applied in the Final Focus, the outcome of this exercise and some problems encountered.

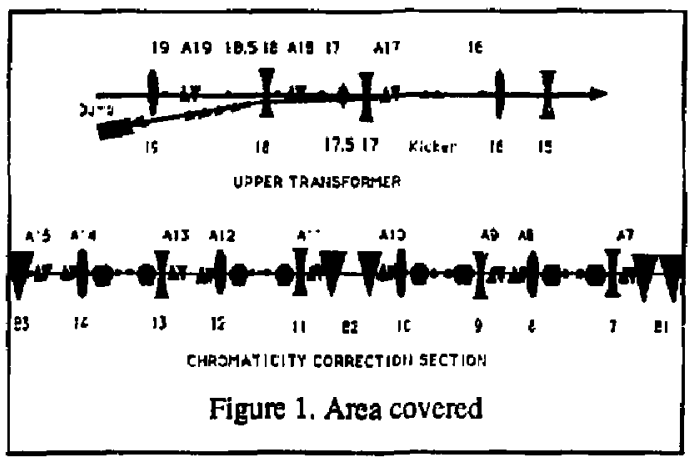

* Supported by DOE under contract \# DE--AC03-76SFO0515

\section{THE SLC FINAL FOCUS}

Figure 1 shows a beamline layout of the pan of the SLC Final Focus where beam based alignment is applied. The Upper Transformer (UT) consists of a -I transformation where the tean divergence at the IP is controlled. The Chromaticity Correction Section (CCS) consists of two -I transformations where the chromaticity and dispersion at the IP can be fine anned. Not shown are two sets of collimators PC18 and PC 18.5 in the Upper Transformer where collimator apertures are tight. The steering effect caused by misaligned quadrupoles in the CCS will in turn lead to spurious focusing and coupling errors due to the sextupoles. It also compromises ure valuable range of the $C C S$ coxtectors which are responsible for conecting the dispersion at the IP. To guarantee the exactness of the $-I$ transformation all the 8 quadrupoles in the CCS are powered in series (Figure 2), which makes an element-by-element analysis of the quadrupole misalignments very difficul. Instead the effects of misaligned clements on the orbit is accumulated over an extended section, the individuat effects being unfolded only in the end via offline analysis.

\section{METHOD AND UNDERL YING PRINCIPLE}

\section{A. Collimators}
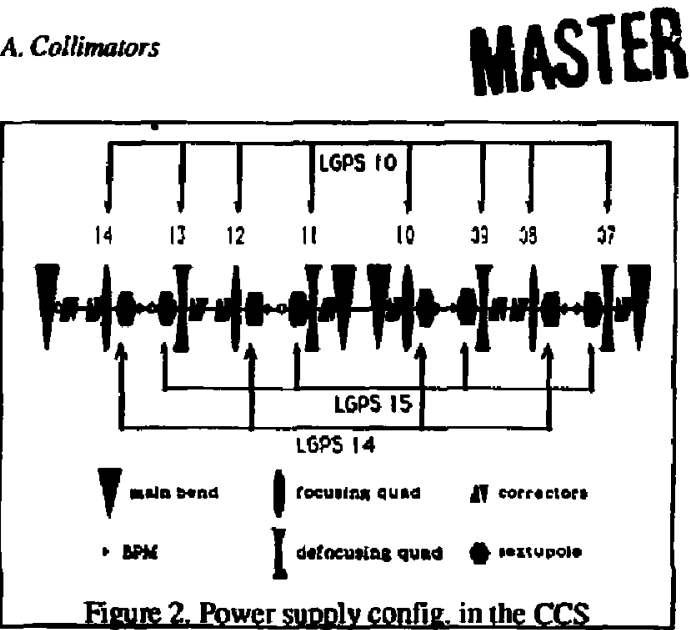

Presented at the IEEE Particle Accelerator Conference, Sar Francisco, CA, May 6-9, 1991. 


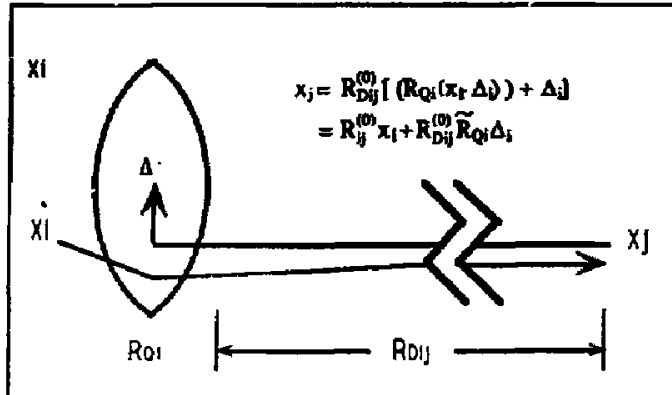

Figure 3. Effect of one misaligred element

The alignment of the collimators PC18 and PC18.5 is done by sweeping ihe beam with upstream correctors overa wide range and recording the ion chamber readings downstream in order to locate the center of the collimator gap. Beam trajectory relative to the collimators can then beconstructed based on these data laver.

\section{B. BPM"s}

In both the UT and the CCS reference lines are constructed by passina a well collimated beam (with smatl energy spread if yossible) through the section in question while all the magnets except the main bends are turned off. The BPMreadings thus obtained are subject to a linear fit to determine the ideal line preferred by the long array of BPM's. Sometimes this line is alternatively determined by the upstream optical condition, Once this reference line is established, all residual BPM readings are incorporated into the database to make them appear aligned along this line. This so-called ballistic study is simple and therefore less susceptible to uncertainties about the beamline. It is a routine practice in the SLCFinal Focus thatresults from other igpes of alignment measurements be checked against the ballis-

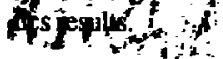

\section{Quadrapoles and Quadrupole Strings}

From the power supply configuration shown in Figure 2 it isclear that we need a general solution for problems involving an array of misaligned optical elements whose compounded effect on the orbit cannot be disentangled on an element-byelement basis. consider an amangement in Figure 3 where a linear optical element has an offset $\Delta i$ with respect to the reference beamline. Here $\Delta \mathrm{i}=\left[\Delta x, \Delta x^{\prime}, \Delta y, \Delta y^{\prime}, \Delta \delta\right]$ and is simply the discrepancy between the nominal beamline and that exnected by the misaligned element. When only linear of fsetsare the concern, only $\Delta x$ and $\Delta y$ are nonzero. The ret effect of the offset in Qi on the orbit is an additional term Bij $\Delta \mathrm{i}$ :

$$
\begin{aligned}
& x_{j}=R_{i j}^{(0)} x_{i}+R_{D i j}^{(0)} \widetilde{R}_{Q_{i}} \Delta_{i} \\
& =R_{i j}^{(0)} x_{i}+B_{i j} \Delta_{i} \\
& \tilde{R}_{Q_{i}} \Delta_{i}=\left\{\begin{array}{l}
\left(I-R_{Q i}\right) \text { for linear offsels } \\
\Delta x_{i}^{\prime} \cdot \theta_{i} \text { for dipole rolls }
\end{array}\right.
\end{aligned}
$$

The above formulation can be exterded to deal with the misalignment effects of an array of linear optical elements. For such a system as shown in Figure 4, the overail effect due to misaligned optical elements can te written as:

$$
\begin{aligned}
& x_{j}^{c}=R_{1 j}^{c(0)} x_{j}^{c}+\sum_{k=1}^{n} T_{k j} \Delta_{k}+\Delta_{j}+\sum_{l=1}^{m} K_{l j}^{c} s_{l} \\
& T_{k j}^{c}=R_{k+1, j}^{c(0)} B_{k, k+1}^{c}, K_{l j}^{c}=R_{l j}^{c(0)} \Delta x_{1}^{c}{ }^{c(0)} \\
& B_{k, k+1}^{c}=\left\{\begin{array}{l}
R_{k, k+1}^{c(0)}\left(I-R_{(k)}^{\left.f_{k}\right)}\right. \text { for linear offsets } \\
R_{k, k+1}^{c(0)} \Delta x_{B k}^{c} c_{k} \text { for dipole rolls }
\end{array}\right.
\end{aligned}
$$

where $\Delta \mathrm{k}$ and $\Delta \mathrm{j}$ stand for the offsets of the optical elements and the BPM's respectively and we have included the possible dipole correctors with their scaling factors being variables. This formulation enables us to treat the system in the SLC Final Focus as described earlier.

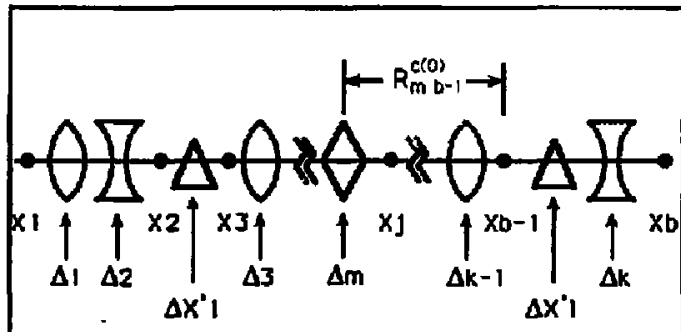

Figure 4. Effect of misaligmed array of elements

Therearejndeed limitations to thismethod. Fundamentally there is no way one can distinguish an unknown deflecting force in the beamline from a real kink in the beam line consistent with the orbil propagation pattern generated by this deflection. This implies that, for example, if a systematic energy error is present, it cannot be distinguished from a real BPM offset pattern consistent with the dispersion function. In such cases the conclusion about misal ignments must be localized ta regions free of intervening bending magnets.

\section{EXPERIENCE WITH THE FINAL FOCUS}

In the SLC Final Focus we use a systematic alignment package to locate the magnet and BPM offsets based on the concept described in Section III. It also accounts for dispersion 


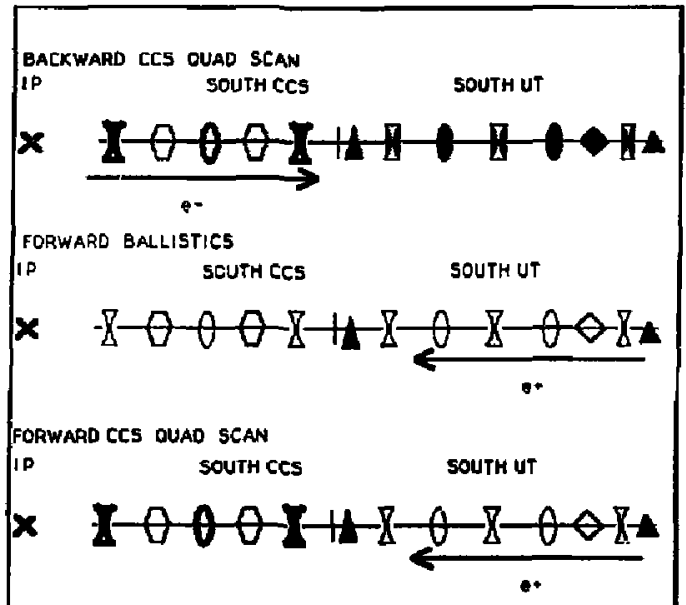

Figure 5. Procedure for CCS quad alignment

fucuration and coupling in the beamline. In the CCS all 8 main quadrupoles are ramped in unison while beam orbits are recorded. Toenhance the accuracy of the measurement, electrons and positrons are brought through the CCS in opposite directions and independenl datasets are taken. The entire procedure is summed up in Figure S. In practice it generally takes two or more iterations of alignment data acquisition and actual moves to converge onto the desired result. The limilation due wenergy/ bend strength mismatch forced us to confine our analysis to relative offsets within regions free of intervening bend magnets. In future aligament experiments we expect to overcoifie this difficulty by incorporating extra monitoring devices such as the spectrometer.

Figure 6(a) shows the alignment situation in the end of the north CCS $x$ plane during the 1990 SLC nun. The data was taken by passing electron beans through the north CCS while the quadrupole string was ramped in steps. Figure $6(b)$ shows the outcome of the sane measurement except that positron beams from the opposite direction were used. Both data sets point to the same offset pattem of the last 4 BPM's (dashed lines) and the last 3 quadrupoles (solid lines). This was confirmed by . the unnatural corrector strengths required in this region for good steering. Movements based on this analysis were implemented which completely relieved the overloaded correctors.

\section{CONCLUSION}

We have established a generalized technique for dealing with linearoptical element of isets in acomplicated beanline. It has been tested in the SLC Final Focus and proved to be successful. A cerain limitation to the melhod such as uncer-

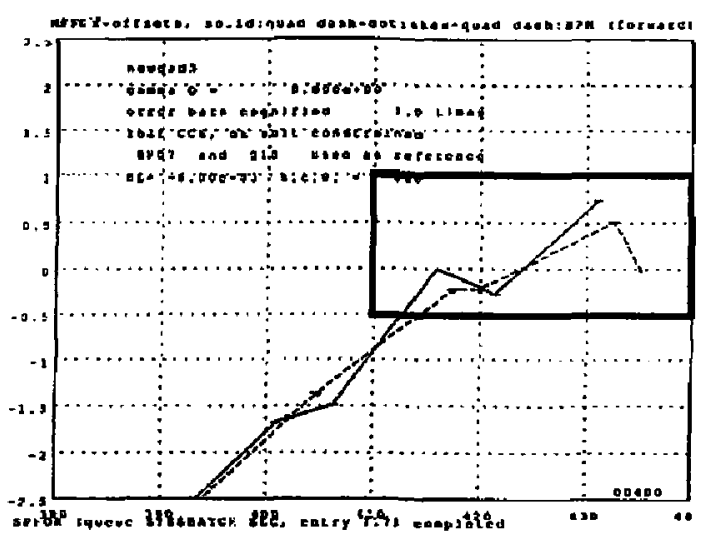

Figure 6(a). CCS $x$-offsets using forward electrons

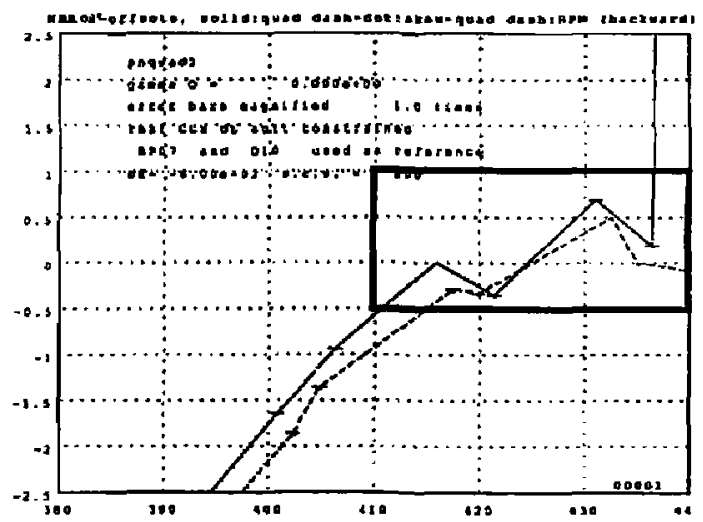

Figure $6(b), \operatorname{CCS} x$-offsets using backward positrons 